\title{
Coupled plasmon-exciton induced transparency and slow light in plexcitonic metamaterials
}

\author{
Panahpour, Ali; Silani, Yaser; Farrokhian, Marzieh; Lavrinenko, Andrei; Latifi, Hamid
}

Published in:

Optical Society of America. Journal B: Optical Physics

Link to article, DOI:

10.1364/JOSAB.29.002297

Publication date:

2012

Document Version

Publisher's PDF, also known as Version of record

Link back to DTU Orbit

Citation (APA):

Panahpour, A., Silani, Y., Farrokhian, M., Lavrinenko, A., \& Latifi, H. (2012). Coupled plasmon-exciton induced transparency and slow light in plexcitonic metamaterials. Optical Society of America. Journal B: Optical Physics, 29(9), 2297-2308. https://doi.org/10.1364/JOSAB.29.002297

\section{General rights}

Copyright and moral rights for the publications made accessible in the public portal are retained by the authors and/or other copyright owners and it is a condition of accessing publications that users recognise and abide by the legal requirements associated with these rights.

- Users may download and print one copy of any publication from the public portal for the purpose of private study or research.

- You may not further distribute the material or use it for any profit-making activity or commercial gain

- You may freely distribute the URL identifying the publication in the public portal 


\title{
Coupled plasmon-exciton induced transparency and slow light in plexcitonic metamaterials
}

\author{
Ali Panahpour, ${ }^{1}$ Yaser Silani, ${ }^{1}$ Marzieh Farrokhian, ${ }^{1}$ Andrei V. Lavrinenko, ${ }^{2}$ and Hamid Latifi ${ }^{1, *}$ \\ ${ }^{1}$ Laser and Plasma Research Institute, Shahid Beheshti University, Evin, 1983963113 Tehran, Iran \\ ${ }^{2}$ DTU Fotonik-Department of Photonics Engineering, Technical University of Denmark, Ørsteds pl. 343, \\ DK-2800 Kongens Lyngby, Denmark \\ ${ }^{*}$ Corresponding author: latifi@Cc.sbu.ac.ir
}

Received February 22, 2012; revised June 30, 2012; accepted July 7, 2012; posted July 9, 2012 (Doc. ID 163430); published August 3, 2012

\begin{abstract}
Classical analogues of the well-known effect of electromagnetically induced transparency (EIT) in quantum optics have been the subject of considerable research in recent years from microwave to optical frequencies, because of their potential applications in slow light devices, studying nonlinear effects in low-loss nanostructures, and development of low-loss metamaterials. A large variety of plasmonic structures has been proposed for producing classical EIT-like effects in different spectral ranges. The current approach for producing plasmon-induced transparency is usually based on precise design of plasmonic "molecules," which can provide specific interacting dark and bright plasmonic modes with Fano-type resonance couplings. In this paper, we show that classical interactions of coupled plasmonic and excitonic spherical nanoparticles (NPs) can result in much more effective transparency and slow light effects in metamaterials composed of such coupled NPs. To reveal more details of the wave-particle and particle-particle interactions, the electric field distribution and field lines of Poynting vector inside and around the NPs are calculated using the finite element method. Finally, using extended Maxwell Garnett theory, we study the coupled-NP-induced transparency and slow light effects in a metamaterial comprising random mixture of silver and copper chloride ( $\mathrm{CuCl}$ ) NPs, and more effectively in a metamaterial consisting of random distribution of coated NPs with $\mathrm{CuCl}$ cores and aluminum shells in the UV region. (c) 2012 Optical
\end{abstract} Society of America

OCIS codes: $\quad 160.3918,260.2030,260.2065,290.4020$.

\section{INTRODUCTION}

Hybrid structures composed of molecular excitons or excitonic semiconductor quantum dots, coupled to metallic nanoparticles have been greatly interested and investigated in recent years, due to their potential applications in the development of functional materials, nanoscale optical devices, molecular sensors, and other applications in biophotonics and nanoplasmonics [1-8]. Novel materials can be assembled from excitonic and plasmonic NPs, joined with biolinkers [4-8]. The metallic NP constituents in such hybrid nanostructures can support localized surface plasmon resonances, providing spatially confined, intensive electric fields on the surface of the NPs. This enhanced near field can strongly modify the properties of nearby atoms or molecules. The coupling between the continuum plasmonic excitations of the resonant metallic NPs and the strong discrete excitations of semiconductor quantum dots (excitons) can lead to interesting effects, such as linear and nonlinear Fano couplings in weak and strong interaction regimes, usually described by quantum mechanical approaches [능] .

If we consider metallic and excitonic NPs as fundamental elements of composite metamaterials, which are macroscopically homogenized, then the size of NPs should be much smaller than wavelength in the spectral range of interest. However, the size of the inclusions can be large enough to be described classically with some proper dielectric functions and polarizabilities. Classical approaches and numerical simulations of Maxwell's equations have been successfully used to model both the optical response of the constituent elements and the collective behavior of many metamaterial nanostructures, containing metallic and semiconductor inclusions [ㅁ-11].

In this paper, we study the classical wave-particle and particle-particle interactions in coupled plasmonic and excitonic spherical NPs as fundamental elements of optical metamaterials, exhibiting electromagnetic transparency and slow light effects. In Section 2 , we study the wave-particle interactions based on Mie scattering theory to calculate the uncoupled polarizabilities and then coupled dipole approximation is used to account for mutual interactions of the NPs. We show that some interesting features can arise, such as enhanced absorption, coupling-induced transparency, and even amplification of the incident wave by individual coupled NPs. We are mostly concerned with Fano-type coupling of the NPs, which can lead to asymmetric absorption profiles and electromagnetic transparency of the coupled NPs. To study the details of the interactions, finite element simulations are performed to calculate the electric field, absorption loss, and power flow lines inside and around the NPs. In Section 3, using extended Maxwell Garnett theory, we study the optical properties of a random mixture of excitonic and plasmonic spherical NPs and also a mixture of spherical coated NPs with excitonic cores and plasmonic shells and show that such coupled plasmonic-excitonic (plexcitonic [8]) core-shell NPs can be considered as building blocks of optical metamaterials, exhibiting transparency and slow light effects. A brief summary is also presented in the last section. 


\section{CLASSICAL INTERACTIONS IN A PLEXCITONIC PAIR}

We consider a pair of coupled spherical silver and copper chloride $(\mathrm{CuCl})$ NPs with equal radius of $a$ and separation of $R$, excited by an electromagnetic plane wave. The mutual interaction of the NPs is studied using coupled dipole approximation and it is supplemented with numerical simulations, which account for higher-order multipolar interactions. We denote the positions and uncoupled polarizabilities by $\mathbf{r}_{j}$ and $\alpha_{j}$, respectively. The induced dipole $\mathbf{p}_{j}$ in each NP, in presence of an applied electric field is $\mathbf{p}_{j}=\varepsilon_{0} \varepsilon_{h} \alpha_{j} \mathbf{E}_{\text {loc } j}(j=1,2)$, where the local field at each NP position $\left(\mathbf{E}_{\mathrm{loc}, j}\right)$ is a sum of the external field and also contribution from the other dipole:

$$
\mathbf{E}_{\mathrm{loc}, j}=\mathbf{E}_{0} \exp \left(i \mathbf{k} \cdot \mathbf{r}_{j}\right)+\mathbf{E}_{\text {dipole }, i \neq j}, \quad(i, j=1,2) .
$$

Here, $E_{0}$ and $k=2 \pi / \lambda$ are the respective amplitude and wave vector of the incident wave. The electric field produced by each dipole is obtained from,

$$
\mathbf{E}_{\text {dipole }, j}=\left[(1-i k r) \frac{3 \hat{\mathbf{r}} \cdot \mathbf{p}_{j} \hat{\mathbf{r}}-\mathbf{p}_{j}}{r^{3}}+k^{2} \frac{\mathbf{p}_{j}-\hat{\mathbf{r}} \cdot \mathbf{p}_{j} \hat{\mathbf{r}}}{r}\right] e^{i k r},
$$

where $\mathbf{r}$ is position vector pointing from dipole to the field point. From these relations, containing the full effects of retardation, the individual coupled polarizabilities and local fields can be calculated.

As a semiconductor with a very sharp excitonic resonance and strong oscillator strength, we consider $\mathrm{CuCl}$ with a dielectric function of the form [9]:

$$
\varepsilon_{p}(\omega)=\varepsilon_{\infty}+A \frac{\gamma}{\omega_{0}-\omega-i \gamma},
$$

where $\varepsilon_{\infty}=5.59$ is the high-frequency limit dielectric constant, $\omega_{0}=5.109 \times 10^{15} \mathrm{rad} / \mathrm{s}$ the exciton resonance frequency, $\gamma=7.596 \times 10^{10} \mathrm{rad} / \mathrm{s}$ the loss factor, and $A=632$, a constant proportional to the exciton oscillator strength. The strength and relaxation of excitonic resonances can only be described quantum mechanically $[\underline{12}, \underline{13}]$, but the response of excitonic materials to light waves can also be described classically by proper dielectric functions such as in relation (3) [14]. In this way, the excitonic resonances are described by very sharp Lorentzian resonances of the dielectric function. The sharp variations in dielectric function of spherical nanoparticles can lead to sharp dipolar and multipolar electric and magnetic Mie resonances, described by Mie coefficients [15]. A classical description of dipolar and multipolar excitations in spherical excitonic nanoparticles and their mutual couplings based on the Mie theory are given in [16].

Since the interactions are studied in a small spectral range around the $\mathrm{CuCl}$ excitonic resonance in the $\mathrm{UV}$ region ( $\lambda_{\text {resonance }} \cong 368.9 \mathrm{~nm}$ ), for the dielectric function of the silver NP, we use a Drude-Lorentz model with two adjusting parameters [17], so that it can be best fitted to the experimental data of Johnson and Christy [18], in the limited spectral range of our interest. The effect of size dependence of damping constant (electron confinement) is also accounted for in this model:

$$
\varepsilon_{\mathrm{Ag}}(\omega, \alpha, \beta)=1-\frac{\omega_{p}^{2}}{\omega(\omega+i \alpha \gamma(a))}+\frac{f \omega_{L}^{2}}{\omega_{L}^{2}-\omega^{2}-i \beta \Gamma_{L} \omega} .
$$

Here, $\omega_{p}=1.39 \times 10^{16} \mathrm{rad} / \mathrm{s}, \omega_{L}=8 \times 10^{15} \mathrm{rad} / \mathrm{s}$, and $\Gamma_{L}=$ $1.73 \times 10^{15} \mathrm{rad} / \mathrm{s}$ are used for silver and adjusting parameters are $\alpha=1.3$ and $\beta=0.2$. The size-dependent plasmonic damping constant is $\gamma(a)=\gamma_{b}+A V_{F} / a$, where $\gamma_{b}=3.19 \times$ $10^{13} \mathrm{rad} / \mathrm{s}$ is the bulk damping constant, $V_{F}$ is the Fermi velocity at the Fermi surface, $a$ is the NP radius, and $A$ is a coefficient equal to 0.25 for spherical particles in vacuum, but can take higher values for particles in a dielectric matrix. We take $A=3 / 4$, which is used by Kreibig [19] for spherical NPs. The real and imaginary parts of the Drude-Lorentz dielectric function curves, fitted to the experimental data of Johnson and Christy for bulk silver are shown in Fig. 1.

Total extinction cross section of the coupled NP pair is calculated from the following relation, which is generally applicable to a group of $N$ interacting particles [20]:

$$
C_{\text {ext }}=\frac{k}{\varepsilon_{0} \varepsilon_{h}\left|E_{0}\right|^{2}} \sum_{j=1}^{N} \operatorname{Im}\left(\mathbf{E}_{\mathrm{inc}, j}^{*} \cdot \mathbf{p}_{j}\right) .
$$

Here, $\mathbf{p}_{j}$ is coupled-induced dipole, calculated from relations (1) and (2). In the simplest case of just two coupled NPs, excited by an incoming plane wave, with electric field vector parallel or perpendicular to the NPs' connecting line, the relation (5) can simply be written in terms of coupled polarizabilities of the NPs:

$$
C_{\text {ext }}=k \operatorname{Im}\left(\sum_{j=1,2} \alpha_{j}^{\text {coupled }}\right) .
$$

We consider two coupled spherical silver and $\mathrm{CuCl}$ NPs with equal radii of $a=10 \mathrm{~nm}$, in a medium with dielectric constant $\varepsilon_{h}=1.6$. The real and imaginary parts of the uncoupled polarizabilities of individual NPs are shown in Fig. 2. We see that the resonance width of the $\mathrm{CuCl} \mathrm{NP}$ is much narrower than that of the silver NP. For $\mathrm{CuCl} \mathrm{NP,} \mathrm{the} \mathrm{strength} \mathrm{of}$ the resonance depends on the exciton oscillator strength, represented by parameter $A$ in relation (3). The width and strength of the resonances for both silver and $\mathrm{CuCl}$ NPs depend also on the sizes of the NPs. Larger diameters lead to lower strengths and also broadening of the resonances due to depolarization effects. The resonance of the $\mathrm{CuCl} \mathrm{NP}$ with $a=10 \mathrm{~nm}$ is mainly dipolar. Using the Mie theory, we can show that the quadrupole moment is negligible except for diameters larger than $40 \mathrm{~nm}$. Figure $\underline{3(\mathrm{a})}-\underline{3(\mathrm{c})}$ show the total

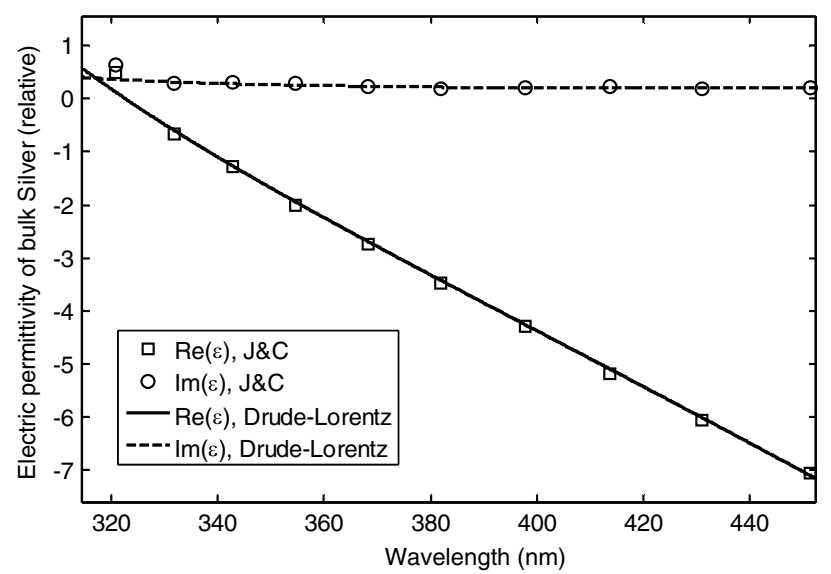

Fig. 1. Real and imaginary parts of the Drude-Lorentz dielectric function curves, fitted to the experimental data of Johnson and Christy for bulk silver. 


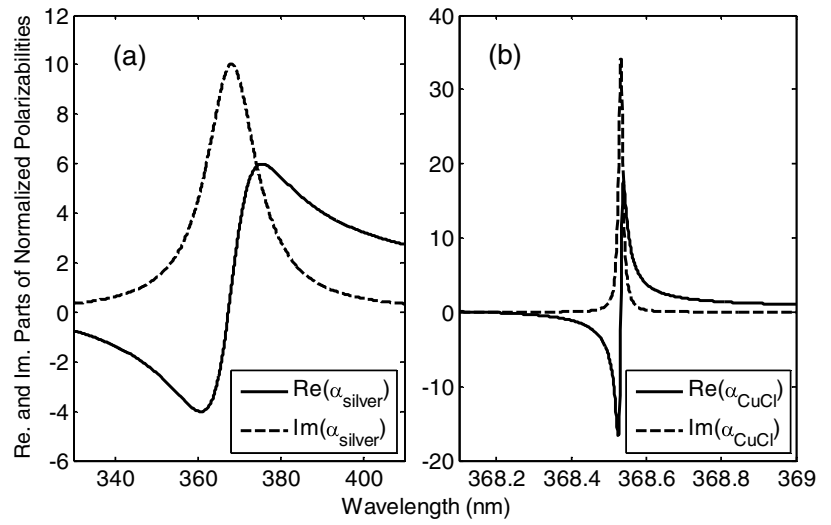

Fig. 2. The real and imaginary parts of the uncoupled polarizabilities of individual silver and $\mathrm{CuCl}$ NPs with equal radii of $a=10 \mathrm{~nm}$, in a medium with dielectric constant $\varepsilon_{h}=1.6$.

extinction coefficients (extinction cross sections normalized to geometrical cross section of the NPs, $\pi a^{2}$ ) of the same coupled silver and $\mathrm{CuCl}$ NPs for different separations of (a) $R=140 \mathrm{~nm}$, (b) $R=80 \mathrm{~nm}$, and (c) $R=50 \mathrm{~nm}$. The incident electric field polarization is assumed to be parallel to the line connecting the NPs' centers. The extinction curves in Fig. 3 have some similarities to the energy absorption curves in weak and strong interaction regimes, described by quantum approaches [5]. In Fig. 3(a), the particles separation is rather large $(R=140 \mathrm{~nm})$ and coupling is accordingly lower, compared to the other curves. In this case the sharp absorption peak of the excitonic resonance is superposed on a much wider extinction curve, corresponding to the localized surface plasmon (LSP) resonance of the silver NP. Figure 3(b) shows the asymmetric Fano coupling of the two resonances, as a result of reduction in NPs separation $(R=80 \mathrm{~nm})$ and consequently improvement of their mutual coupling. We can see both enhancement and suppression of absorption around the resonance line of the $\mathrm{CuCl} \mathrm{NP}$. As shown in Fig. 3(c), by further increasing the NPs' coupling (reducing the separation to $R=50 \mathrm{~nm}$ ) the excitonic resonance produces a narrow transparency dip within the wider plasmonic extinction curve and the redshift of the LSP resonance is further increased. The curves show that the extinction profile of the pair is quite sensitive to the NPs' coupling strength. The respective Figs. $\underline{4}$ and $\underline{5}$ show the increase of the depth and

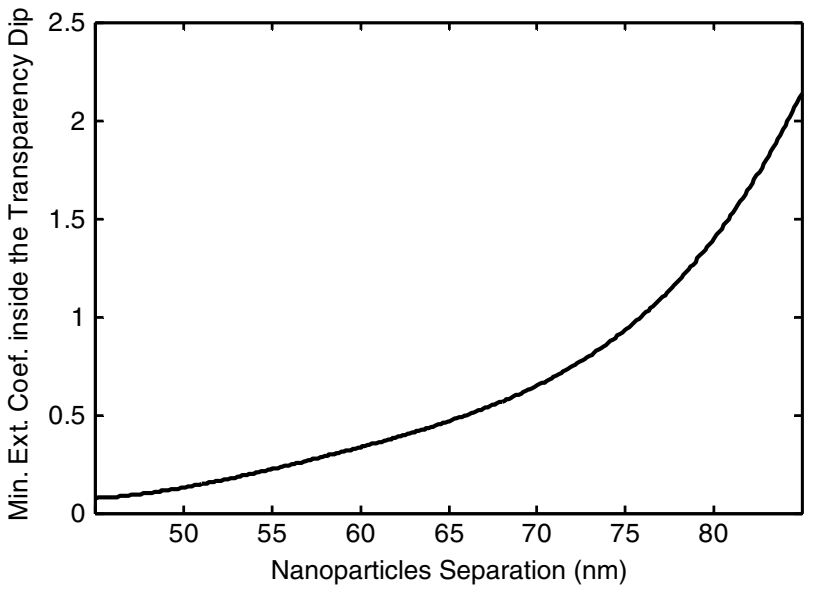

Fig. 4. The minimum values of extinction coefficient inside the transparency dip, produced by two coupled silver and $\mathrm{CuCl} \mathrm{NPs} \mathrm{with} \mathrm{the}$ same parameters as in Fig. 3 , as a function of separation of the NPs.

width of the transparency dip for larger couplings or smaller separations. Also, the redshift of the transparency dip as a function of the separation of the NPs is shown in Fig. 6 . The redshift is calculated from the reference wavelength related to the case where the separation of the NPs is $R=$ $40 \mathrm{~nm}$. Furthermore, it can be seen that for larger frequency separations between the excitonic and plasmonic resonances, the transparency is more pronounced and the width of the dip is increased.

For the same NPs corresponding to Fig. 3(a), the extinction coefficient of the individual coupled silver NP is plotted in Fig. 7. We see that while the total extinction of the pair in Fig. 3 (a) shows a very sharp excitonic peak over the wide LSP curve, the contribution of the coupled silver NP is an LSP extinction profile with a quite narrow dip in the middle, approaching to zero at the bottom. For providing more clarity, the overall extinction coefficient (solid curve) and the contributions from individual coupled $\mathrm{CuCl}$ (dotted curve) and silver NPs (dashed curve) are plotted in Fig. 8, in a much narrower spectral range of $\lambda=368.51-368.63 \mathrm{~nm}$. The curves show that for $R=140 \mathrm{~nm}$, while the $\mathrm{CuCl} \mathrm{NP}$ and the whole plexcitonic pair are highly absorptive, the silver NP is nearly transparent to the incident wave in the small wavelength range inside the narrow dip.
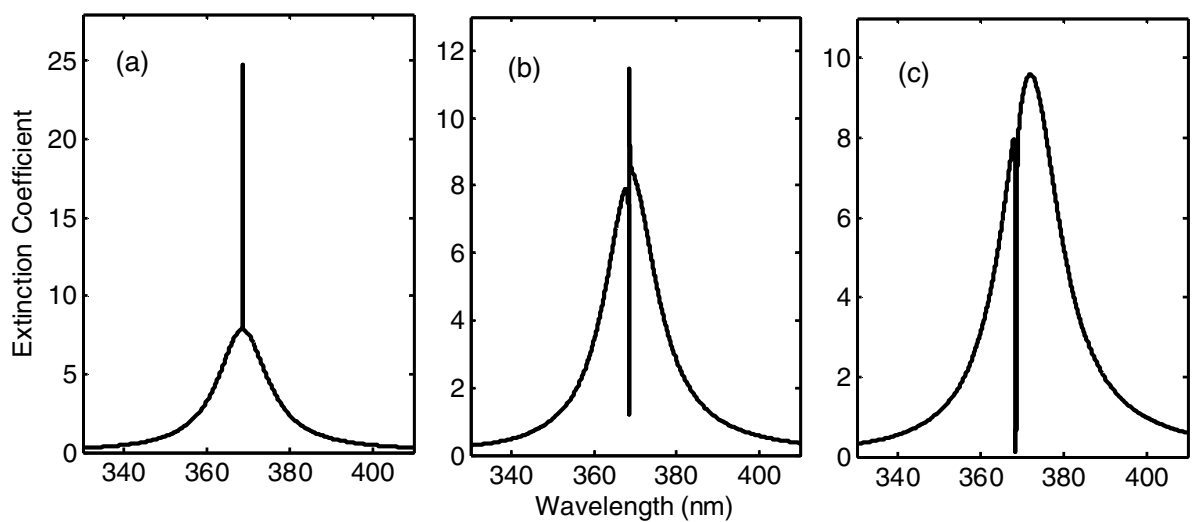

Fig. 3. Total extinction coefficients of two coupled spherical silver and $\mathrm{CuCl}$ NPs with equal radii of $a=10 \mathrm{~nm}$, in a medium with dielectric constant $\varepsilon_{h}=1.6$, for different separations of (a) $R=140 \mathrm{~nm}$; (b) $R=80 \mathrm{~nm}$, and (c) $R=50 \mathrm{~nm}$. The incident electric field polarization is assumed to be parallel to the line connecting the NPs. 


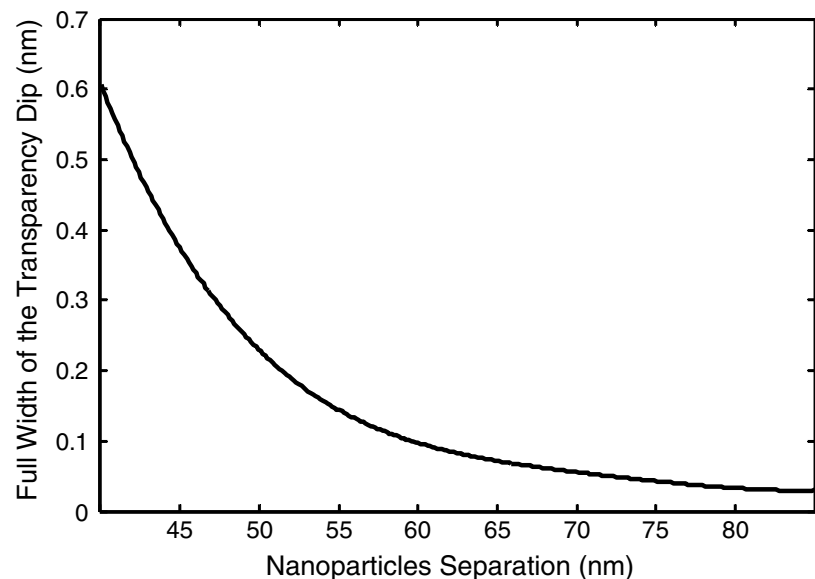

Fig. 5. Full-width of the transparency dip as a function of separation of two coupled silver and $\mathrm{CuCl}$ NPs with the same parameters and conditions as in Fig. 3.

As shown in Figs. 3(b) and 3(c), corresponding to smaller separations of the $\overline{\mathrm{NPS}}$ (or larger couplings), considerable suppression is produced in the total extinction of the pair near the sharp excitonic resonance of the $\mathrm{CuCl} \mathrm{NP}$, which means that the pair as a whole can also be nearly transparent to the incident wave in a narrow wavelength range. Within the transparency windows produced in the extinction profiles of the plexcitonic pairs in Figs. 3(b) and 3(c), the overall polarizability of the NPs shows steep variations, which can produce strong normal dispersions in media containing large number of such NPs. The real part of the total polarizability (normalized to $4 \pi a^{3}$ ) of the same NP pair of Fig. 3(c) for $R=50 \mathrm{~nm}$ is plotted in Fig. 9. The steep variation of the polarizability near the excitonic resonance, together with the very low extinction coefficient of the NP pair in the same spectral range [Fig. 3(c)], suggests that plexcitonic pairs can be considered as building blocks of composite metamaterials exhibiting transparency and slow light effects. In Section 3 , we show that a random mixture of such interacting NPs can show such effects.

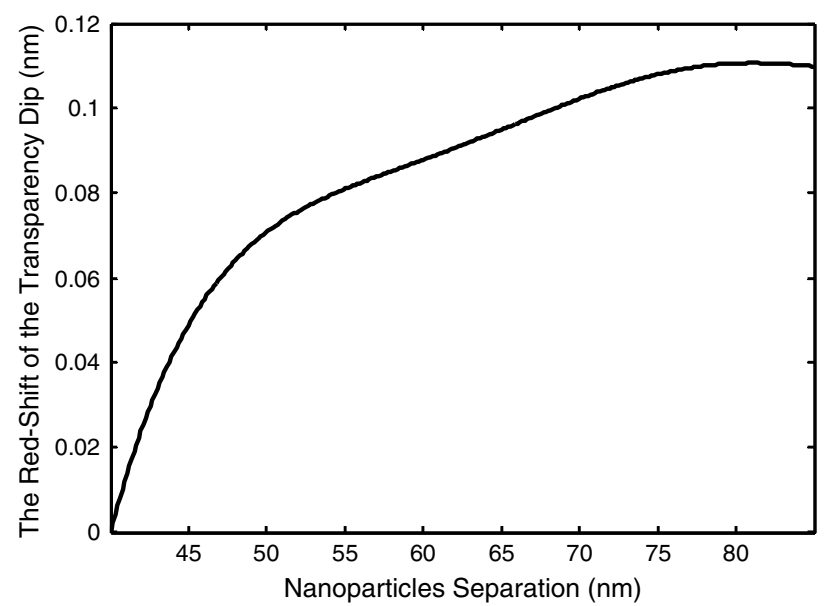

Fig. 6. The redshift of the minimum point of the transparency dip as a function of separation of two coupled silver and $\mathrm{CuCl}$ NPs with the same parameters and conditions as in Fig. 3. The redshift is calculated from the reference wavelength related to the case where the separation of the NPs is $R=40 \mathrm{~nm}$.

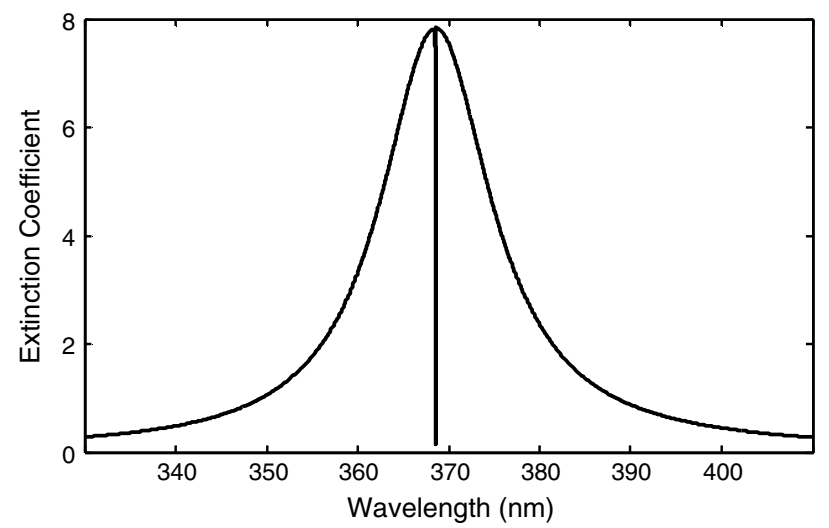

Fig. 7. Extinction coefficient of the silver NP in a plexcitonic pair with the same parameters and conditions as in Fig. 3(a).

The conventional methods of plasmon-induced transparency as classical analogues of the quantum optical effect of EIT [21] are usually based on designing specific plasmonic "molecules" for providing two interacting superradiant and subradiant plasmonic modes [22-26]. The superradiant mode (or the "bright" state) in such synthetic molecules is directly excited by the external field and the subradiant mode ("dark" state) is excited through coupling to the superradiant mode. The transparency effect results from the destructive interference between two different excitation pathways; one, being the direct excitation of the bright mode by the incident wave and the other, excitation through coupling to the dark mode. The destructive interference of the two excitation pathways leads to the development of a dip in the imaginary part of the polarizability of the bright mode and steep variation of the polarizability in the same spectral region. The plasmoninduced transparency methods are different from our proposed approach, based on coupling of NPs in plexcitonic molecules. In a plexcitonic molecule, both of the NPs have strong couplings to the external field. In addition, the resonance of the excitonic NP is much sharper than the dark-state plasmonic resonance, which suffers from ohmic losses inherent in metals. Therefore, the transparency effect can be much more efficient and pronounced in a plexcitonic molecule than a plasmonic one. Plasmonic molecules usually lead to group

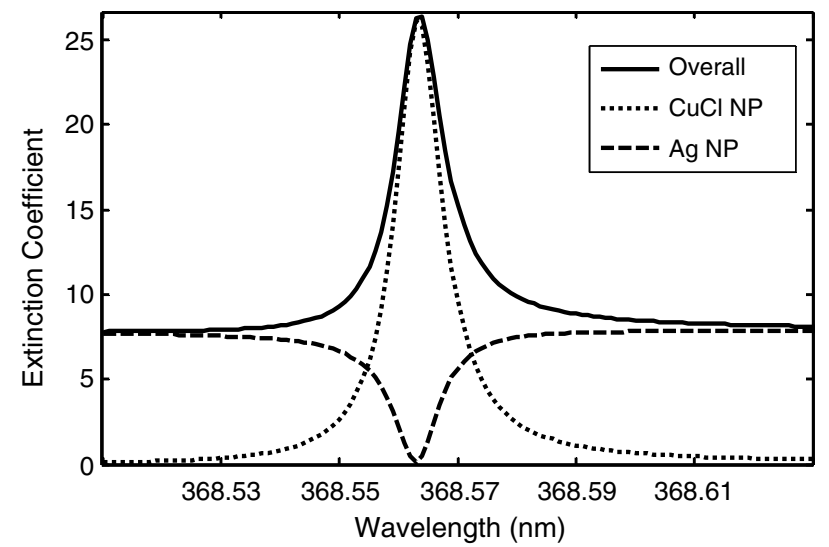

Fig. 8. The overall extinction coefficient (solid curve) and the extinction contributions from $\mathrm{CuCl}$ (dotted curve) and silver NPs (dashed curve) in a plexcitonic pair with the same parameters and conditions as in Fig. 3(a). 


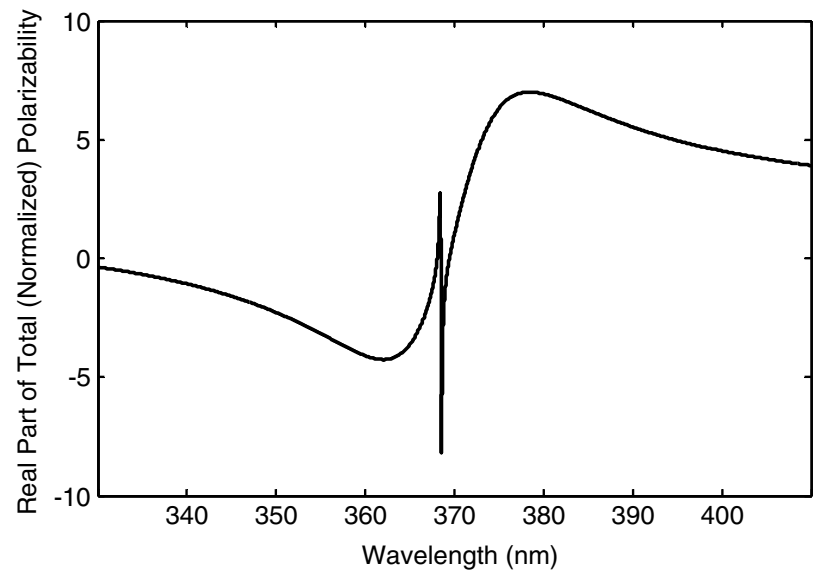

Fig. 9. Real part of total polarizability (normalized to $4 \pi a^{3}$ ) of the same NP pair of Fig. $3(\mathrm{c})(R=50 \mathrm{~nm})$.

indices of refraction of the order of several hundreds [22-26], while as we show in the following sections, group indices in the proposed plexcitonic metamaterials can be of the order of several thousands.

The enhancement and suppression of extinction cross sections are not the only features arising from mutual interaction of excitonic and plasmonic NPs. In addition to zero extinction or transparency, we show that in proper conditions, each coupled NP in a plexcitonic pair can even exhibit negative extinction or amplification in a specific wavelength range. We consider the extinction of a plane wave by two coupled silver and $\mathrm{CuCl}$ NPs in the same condition of Fig. $\underline{3(\mathrm{c}) \text {. Figure } 10}$ shows the contribution of individual coupled NPs to the extinction coefficient, separately. We see that for each NP, there is a narrow negative extinction region, near the $\mathrm{CuCl}$ resonance. For the same NPs, the contribution of individual NPs to the extinction and also the total extinction coefficient of the pair are plotted in Fig. 11, in a much narrower wavelength range, showing that both silver (dashed curve) and $\mathrm{CuCl}$ (dotted curve) NPs exhibit amplification in the respective wavelength ranges of $\lambda \cong 368.51-368.55 \mathrm{~nm}$ and $\lambda \cong$ $368.2-368.51 \mathrm{~nm}$. However, the sum of the two curves corresponding to the overall extinction of the NPs (the solid curve) is always positive, so the system as a whole is absorptive. We note that the NPs' amplifications cannot be observed in far

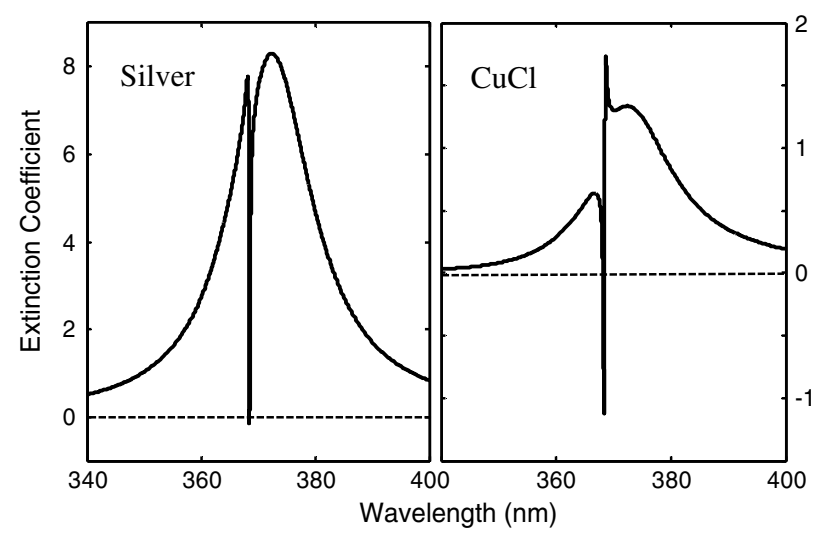

Fig. 10. The contribution of individual coupled silver and CuCl NPs to the extinction coefficient, in a plexcitonic pair, with the same parameters and conditions as in Fig. 3(c).

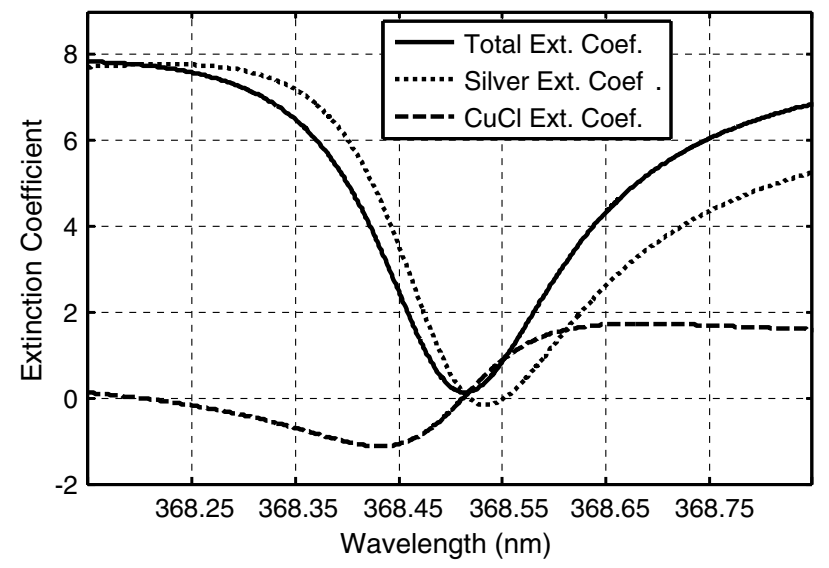

Fig. 11. Total extinction coefficient (solid curve) of the same plexcitonic pair as in Fig. 10 and the contribution of individual NPs to the extinction (dashed and dotted curves).

field, because what we observe in far field is an overall effect, which is a positive extinction.

The physics of the classical plasmon-exciton interactions can be better understood if we consider the two NPs as two classical oscillators with uncoupled polarizabilities of Lorentzian form, and then instead of relation (2) we assume a much more simpler relation for the electric field produced by each dipole, $\mathbf{E}_{\text {dipole } j}=C(R) \cdot \mathbf{p}_{j},(j=1,2)$, where $C(R) \propto 1 / R^{3}$, and $R$ is the distance between the NPs. We assume that the NPs are excited by a plane wave with the electric field amplitude denoted by $E_{0}$ and wavelength much larger than the NPs' sizes and separation. So, the induced dipole moment in each of the NPs can be written as

$$
\begin{gathered}
P_{1}=\alpha_{1}\left(E_{0}+C P_{2}\right), \\
P_{2}=\alpha_{2}\left(\beta E_{0}+C P_{1}\right) .
\end{gathered}
$$

The parameter $\beta$ is inserted in relation (8) in order to consider two different cases. When both of the NPs are effectively coupled to the external field, we put $\beta=1$ and when the excitation of the second NP is mainly through coupling to the other NP we put $\beta=0$. The relations ( $\underline{7})$ and (ㅁ) can be solved for the induced dipole moments:

$$
P_{j}=\alpha_{j}^{\prime} E_{0}, \quad j=1,2
$$

with coupled polarizabilities of

$$
\begin{aligned}
& \alpha_{1}^{\prime}=\alpha_{1} \frac{1+\beta C \alpha_{2}}{1-C^{2} \alpha_{1} \alpha_{2}}, \\
& \alpha_{2}^{\prime}=\alpha_{2} \frac{\beta+C \alpha_{1}}{1-C^{2} \alpha_{1} \alpha_{2}} .
\end{aligned}
$$

We assume that the uncoupled polarizabilities have a Lorentzian response:

$$
\alpha_{j}=\frac{A}{\omega_{j}^{2}-\omega^{2}-i \omega \gamma_{j}}, \quad j=1,2,
$$


where $\omega_{j}$ and $\gamma_{j}$ are the center frequency and damping constant of the Lorentzian polarizability $\alpha_{j}$, and $A$ is a constant. Substituting the uncoupled polarizabilities $\left(\alpha_{j}, j=1,2\right)$ into relations (10) and (11), we get

$$
\begin{aligned}
& \alpha_{1}^{\prime}=\frac{\left(\delta_{2}-i \gamma_{2} \omega\right)-\beta C}{\left(\delta_{1}-i \gamma_{1} \omega\right)\left(\delta_{2}-i \gamma_{2} \omega\right)-C^{2}} A, \\
& \alpha_{2}^{\prime}=\frac{\left(\delta_{1}-i \gamma_{1} \omega\right) \beta-C}{\left(\delta_{1}-i \gamma_{1} \omega\right)\left(\delta_{2}-i \gamma_{2} \omega\right)-C^{2}} A,
\end{aligned}
$$

in which $\delta_{j}=\omega_{j}^{2}-\omega^{2}(j=1,2)$. The condition of negligible coupling of the second NP to the external field $(\beta \cong 0)$, corresponds to classical analogy of the Fano resonance and classical EIT, which is thoroughly discussed in [27] and we do not repeat the results here, except for a brief description of the phenomenon. If we assume $\omega_{1} \neq \omega_{2}$ and $\gamma_{2} \ll \gamma_{1}$ in relations (13) and (14), the absolute value of $\alpha_{1}^{\prime}$ as a function of $\omega$, shows two peaks corresponding to the resonances of the two NPs with a minimum between them at a frequency very close to $\omega_{2}$. The minimum of the curve approaches to zero as $\gamma_{2}$ takes smaller values. We can see that at this point the oscillation of the first NP is out of phase with and quenched by the second NP oscillation, which is synchronized with the external field. In this case while the imaginary part of $\alpha_{1}^{\prime}$ is always positive the imaginary part of $\alpha_{2}^{\prime}$ is negative for $\omega<\sqrt{\left(\omega_{1}^{2} \gamma_{2}+\gamma_{1} \omega_{2}^{2}\right) /\left(\gamma_{1}+\gamma_{2}\right)}$.

For the case that both of the NPs are effectively coupled to the external field $(\beta=1)$, we calculate the phase angle and imaginary part of the polarizabilities in (13) and (14) near the resonances. Using the parameters, $A / \omega_{1}^{2}=0.0851$, $\gamma_{1} / \omega_{1}=0.0117, \quad \gamma_{2} / \omega_{1}=1.167 \times 10^{-4}, \quad C / \omega_{1}^{2}=2.735 \times 10^{-4}$, the imaginary part of $\alpha_{1}^{\prime}+\alpha_{2}^{\prime}$ and the phase angles of $\alpha_{1}^{\prime}=$ $\left|\alpha_{1}^{\prime}\right| \exp \left(i \phi_{1}(\omega)\right)$ and $\alpha_{2}^{\prime}=\left|\alpha_{2}^{\prime}\right| \exp \left(i \phi_{2}(\omega)\right)$ are shown in Figs. 12, 13, and 14, for three different values of $\omega_{2} / \omega_{1}=1$, 1.0018 and 0.9982 , respectively. In Fig. 12 , where $\omega_{1}=\omega_{2}$, we see the enhancement of the imaginary part of $\alpha_{1}^{\prime}$ by the sharp peak corresponding to the imaginary part of $\alpha_{2}^{\prime}$ at $\omega=\omega_{1}=\omega_{2}$, where the phases of the resonances are equal

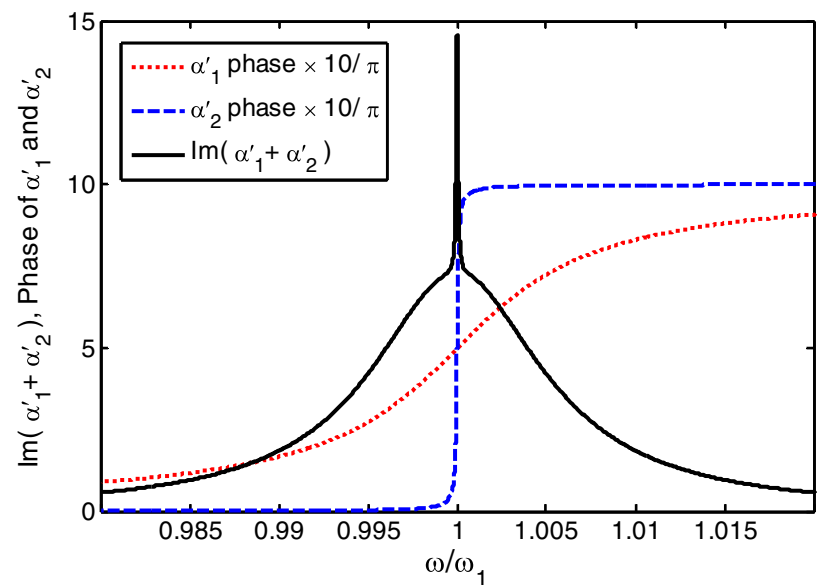

Fig. 12. (Color online) The imaginary part of the total (coupled) polarizabilities, $\alpha_{1}^{\prime}+\alpha_{2}^{\prime}$ and the phase angles of $\alpha_{1}^{\prime}$ and $\alpha_{2}^{\prime}$, for the case that both of the NPs are effectively coupled to the external field $(\beta=1)$ and $\omega_{2}=\omega_{1}$, using the parameters $A / \omega_{1}^{2}=0.0851$, $\gamma_{1} / \omega_{1}=0.0117, \gamma_{2} / \omega_{1}=1.167 \times 10^{-4}, C / \omega_{1}^{2}=2.735 \times 10^{-4}$.

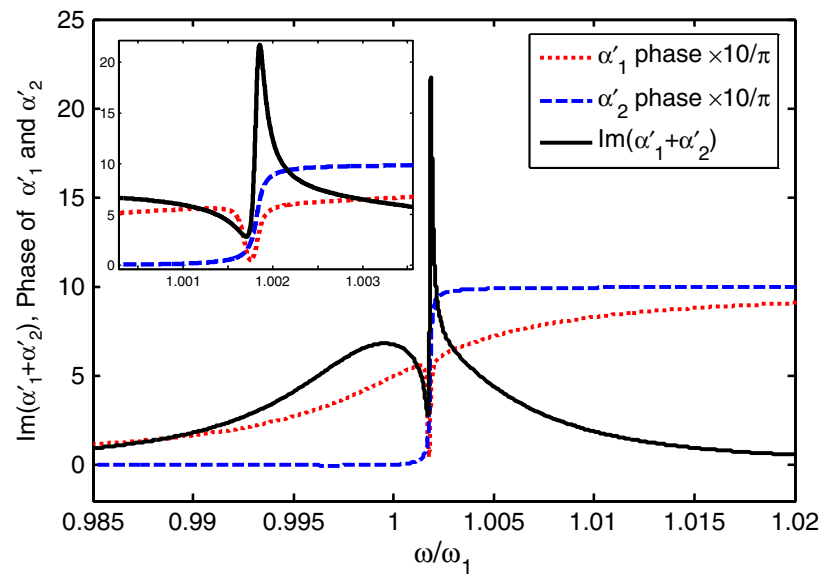

Fig. 13. (Color online) The imaginary part of the total (coupled) polarizabilities, $\alpha_{1}^{\prime}+\alpha_{2}^{\prime}$ and the phase angles of $\alpha_{1}^{\prime}$ and $\alpha_{2}^{\prime}$, for the same NPs and parameters used in Fig. 12, except for the ratio, $\omega_{2} / \omega_{1}=1.0018$. The inset shows a closer view of the curves near $\omega=\omega_{2}$.

to $\pi / 2$. So, at this frequency both of the NPs are effectively coupled to the external field and their amplitudes are added constructively. In Fig. 13, showing the same curves at $\omega_{2}=$ $1.0018 \omega_{1}$, we see the phase angle $\phi_{2}(\omega)$ is nearly the same as that of an uncoupled NP $\left(\alpha_{2}\right)$, but the phase angle $\phi_{1}(\omega)$ at the minimum point of the solid curve shows an abrupt change from $\cong \pi / 2$ toward the zero. The inset shows a closer view of the curves around $\omega=\omega_{2}$. We see that both of the NPs have phase values near to zero at this point, showing that they are not effectively coupled to the external field and are nearly transparent at this frequency. Figure 14 shows similar curves for the same parameters but when $\omega_{2}=0.9982 \omega_{1}$. In this figure also, near the minimum point of the solid curve, we see an abrupt change of $\phi_{1}(\omega)$ from values near $\pi / 2$ toward the zero. This leads to lower coupling of the first NP to the external field. In this figure and Fig. 13, the phase angles corresponding to the maximum points of the solid curves are around $\pi / 2$, showing that in these cases, both of the NPs are highly coupled to the external field.

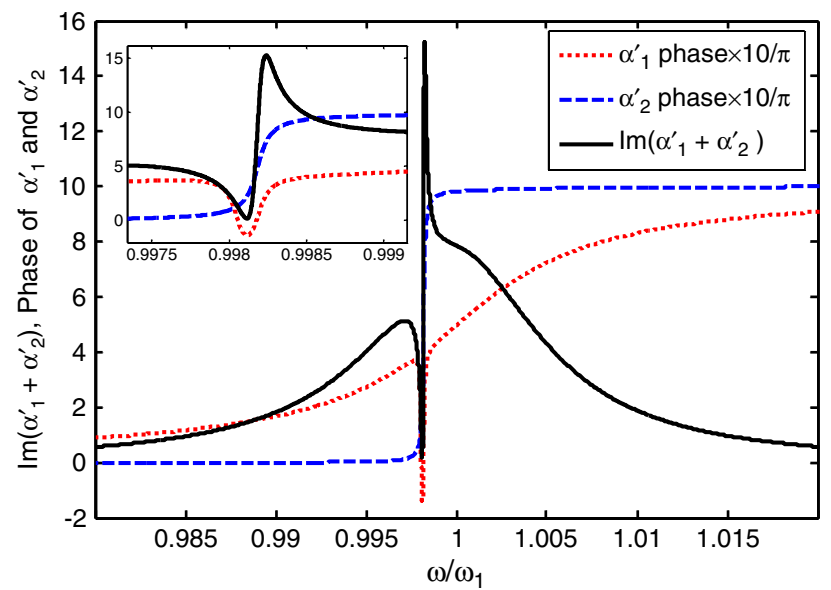

Fig. 14. (Color online) The imaginary part of the total (coupled) polarizabilities, $\alpha_{1}^{\prime}+\alpha_{2}^{\prime}$ and the phase angles of $\alpha_{1}^{\prime}$ and $\alpha_{2}^{\prime}$, for the same NPs and parameters used in Fig. 12, except for the ratio, $\omega_{2} / \omega_{1}=0.9982$. The inset shows a closer view of the curves near $\omega=\omega_{2}$. 
Other features of the two interacting NPs can be revealed through changing the parameters such as coupling coefficient $(C)$ or the difference between the resonance frequencies, $\Delta \omega=\left|\omega_{1}-\omega_{2}\right|$. In general by increasing these parameters, both the depth and width of the produced dip in the imaginary part of $\alpha_{1}^{\prime}+\alpha_{2}^{\prime}$ increases.

It can be shown easily that the imaginary parts of both of the coupled polarizabilities $\alpha_{1}^{\prime}$ and $\alpha_{2}^{\prime}$ can take negative values in different specific frequency ranges, while the sum of the imaginary parts is positive at all frequencies:

$$
\operatorname{Im}\left(\alpha_{1}^{\prime}+\alpha_{2}^{\prime}\right)=\frac{\gamma_{2}\left(C-\delta_{1}\right)^{2}+\gamma_{1} \gamma_{2}\left(\gamma_{1}+\gamma_{2}\right) \omega^{2}+\gamma_{1}\left(\delta_{2}^{2}+C^{2}\right)}{\left(\delta_{1} \delta_{2}-\gamma_{1} \gamma_{2} \omega^{2}-C^{2}\right)^{2}+\left(\delta_{1} \gamma_{2} \omega+\delta_{2} \gamma_{1} \omega\right)^{2}}>0 .
$$

This is another feature of the interacting bright NPs $(\beta=1)$, showing that their overall extinction is always positive, while the individual coupled NPs can take negative extinction coefficients in separate narrow spectral ranges.

More details of the wave-particle and particle-particle interactions can be observed by studying the field lines of Poynting vector inside and around the NPs near their resonances [28] and in the spectral range of exciton-plasmon interaction. In Figs. 15(a) to 15(c), some results of our numerical calculations based on the finite element method are shown, where the power flow lines are plotted in the polarization plane of the incident field, inside and around a single silver NP of radius $a=10 \mathrm{~nm}$ in a medium with dielectric constant $\varepsilon_{h}=$ 1.6 and at different wavelengths of $\lambda=365 \mathrm{~nm}$ [Fig. 15(a)], $\lambda=363 \mathrm{~nm}$ [Fig. 15(b)], and $\lambda=390 \mathrm{~nm}$ [Fig. 15(c)]. The incident plane wave propagates in $+z$ direction and the field polarization is parallel to the $x$ axis. The color legends represent the $z$ component of Poynting vector normalized to the intensity of incident wave. In Fig. 15(a), corresponding to LSP resonance peak of the $\mathrm{NP}$ at the wavelength $\lambda=365 \mathrm{~nm}$, we see how the particle attracts the power flow from an area much larger than its geometrical cross section, so that its extinction coefficient is much larger than unity. Also, we can see that the power intake is from a small surface area on the back side of the NP. Figure 15(b) corresponds to $\lambda=363 \mathrm{~nm}$, a little smaller than the wavelength of LSP resonance peak. It is seen that only part of the entering power from the back side is trapped into the optical whirlpools to be absorbed by the NP, and some part of energy can escape and exit from the front side of the NP. Figure 15(c) corresponds to the wavelength of $\lambda=390 \mathrm{~nm}$, which is rather far from the NP's LSP resonance peak, where the extinction cross section is much smaller than the geometrical cross section. In this figure we see that the trapped power flow comes from an area much smaller than geometrical cross section and the power intake is from an area in front of the NP. Also, the power flow lines rotate in the opposite direction, compared to that in Fig. 15(b). Similar results can also be obtained for a single $\mathrm{CuCl \textrm {NP }}$, at its resonance, and also at wavelengths a little smaller and larger than the resonance line.

For a pair of coupled $\mathrm{CuCl}$ and silver NPs with the same radius of $a=10 \mathrm{~nm}$ and separation of $R=30 \mathrm{~nm}$, in a host medium with dielectric constant $\varepsilon_{h}=1.6$, we numerically calculated the normalized value of electric field at the center of each NP (shown in Fig. 16), which can be considered as a measure of strength of the wave-particle interaction. Also,
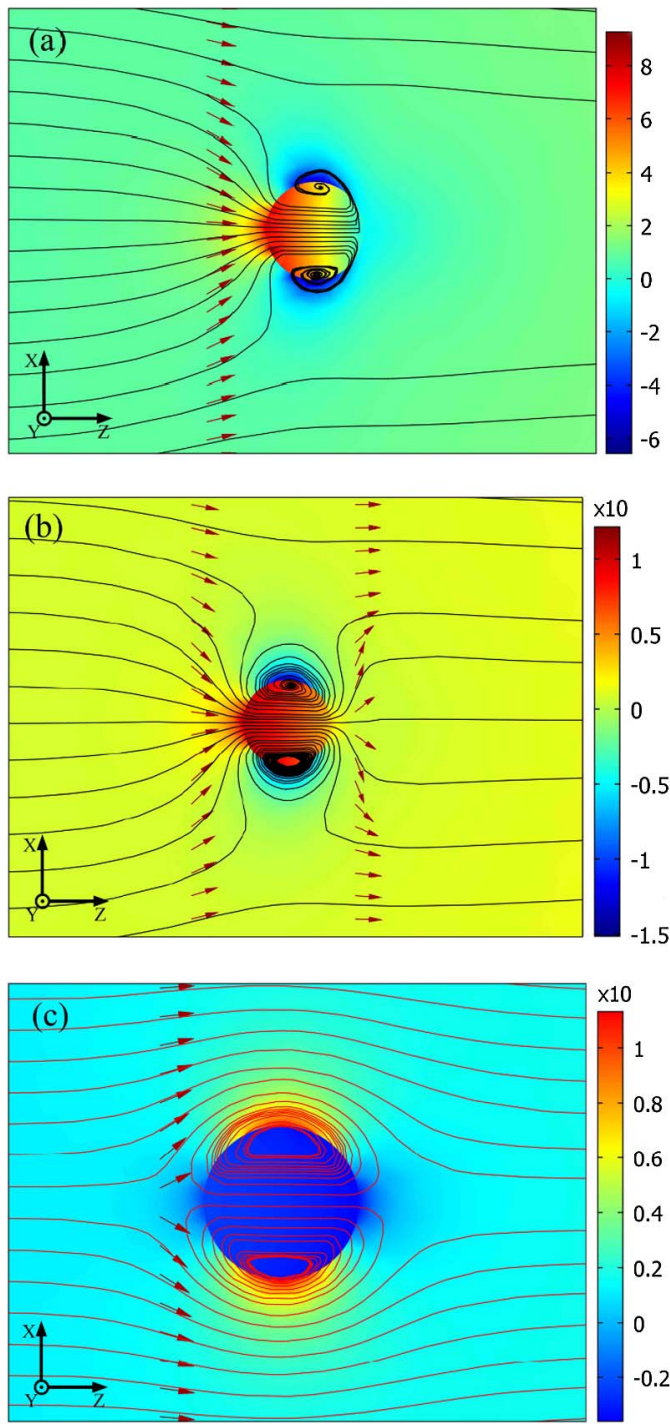

Fig. 15. (Color online) Power flow lines in $x-z$ plane, inside and around a single silver NP of radius $a=10 \mathrm{~nm}$, in a medium with dielectric constant $\varepsilon_{h}=1.6$, at the wavelengths of (a) $\lambda=365 \mathrm{~nm}$, corresponding to the LSP resonance of the NP; (b) $\lambda=363 \mathrm{~nm}$, a little smaller than the wavelength of the NP's LSP resonance peak, and (c) $\lambda=390 \mathrm{~nm}$, far from the wavelength of NP's LSP resonance peak. The plane wave propagation is in $+z$ direction and the field polarization is parallel to the $x$ axis. The color legends represent the $z$ component of the Poynting vector normalized to the incident wave intensity.

the total power loss due to absorption is calculated and shown in Fig. 17. In these simulations the incident plane wave propagates in $+z$ direction, perpendicular to the line connecting the NPs' centers and the field polarization is along the $x$ axis and parallel to the NPs' connecting line. Near the minimum point of total absorption curve in Fig. 17, the power flow lines around the NPs have a typical pattern shown in Fig. 18(a), corresponding to the wavelength of $\lambda=368.55 \mathrm{~nm}$. In this figure, the optical whirlpool between the NPs and passing through both of them visualizes the mutual couplings of the NPs. The color legends in this and in the following figures represent the $x$ component of electric field normalized to the incident field amplitude. Figure $18(\mathrm{~b})$ shows the power flow lines and field distribution at the wavelength of $\lambda=368.56 \mathrm{~nm}$, exactly at the minimum point of the total 


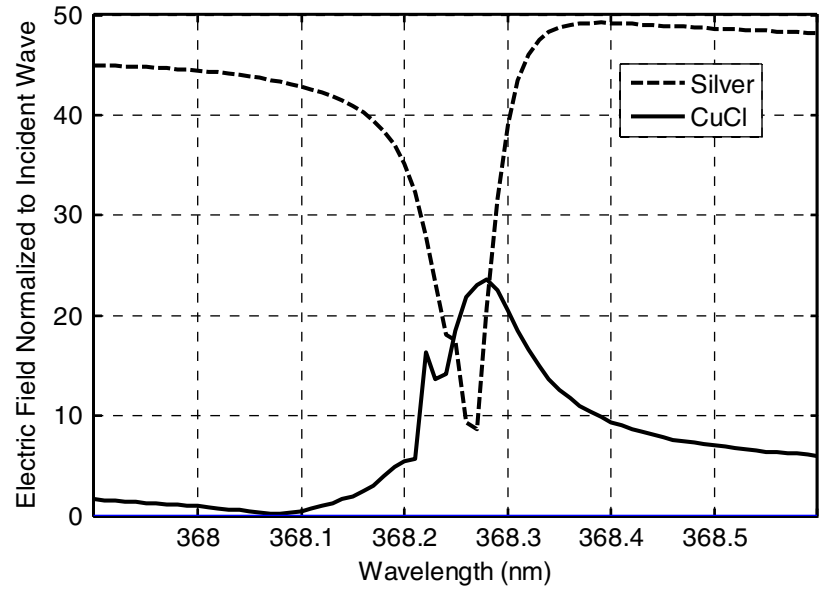

Fig. 16. (Color online) Normalized (to the incident wave amplitude) electric field at the center of each NP in a plexcitonic molecule, composed of two coupled $\mathrm{CuCl}$ and silver NPs with the same radius of $a=10 \mathrm{~nm}$ and separation of $R=30 \mathrm{~nm}$, in a medium with dielectric constant $\varepsilon_{h}=1.6$, calculated by finite element method.

absorption curve in Fig. 17. We see that just a small number of power lines are trapped into the optical whirlpools and most of them easily pass through or turn around the NPs. Figure 18(c), corresponds to the minimum value of electric field (Fig. 16) at the center of the silver NP and at the wavelength $\lambda=368.57 \mathrm{~nm}$. In contrast to Figs. 18(a) and 18(b), where the power flow lines are fed mainly from the front side of the silver NP, in Fig. 18(c) the power intake is from a small surface area on the back side of the silver NP. This is due to an unusual phase change in polarizability of the silver $\mathrm{NP}$, so that the local field at the position of the silver NP, and the wave-particle interactions are minimum at this wavelength. Figure $18(\mathrm{~d})$ is related to the maximum point of total absorption loss at $\lambda=386.63 \mathrm{~nm}$ (Fig. 17). Here we see that power flow pattern for both of the NPs has changed and the power is mainly fed from the front side. This can also be attributed to the phase changes in polarizabilities of the NPs, returning to the value of $\phi \approx \pi / 2$, leading to high absorption losses of the pair at this wavelength.

We note that in Fig. 16, there is a small and rather sharp peak on the left side of the main peak of the electric field at the center of the $\mathrm{CuCl} \mathrm{NP}$. This is due to the electric quad-

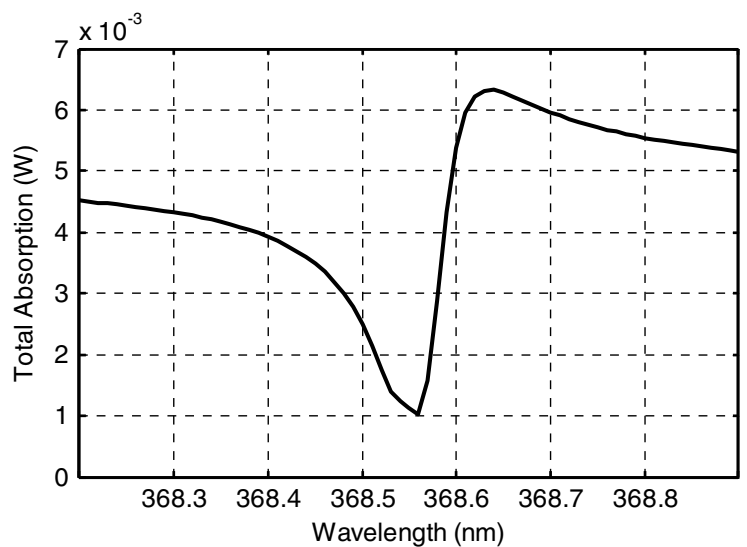

Fig. 17. Total power loss due to absorption, corresponding to a plexcitonic molecule with the same parameters and conditions as in Fig. 16, calculated by the finite element method. rupole component of the field at $\lambda=386.52 \mathrm{~nm}$. As shown in Fig. 18(e), the field distribution around this wavelength and power flow patterns are quite different from previous figures and some smaller scale optical whirlpools are produced near the surface of the $\mathrm{CuCl} \mathrm{NP}$.

\section{TRANSPARENCY AND SLOW LIGHT IN PLEXCITONIC METAMATERIALS}

In the previous sections, we showed that Fano-like coupling of excitonic and plasmonic NPs can render a plexcitonic pair transparent to the incident wave. This suggests that a medium comprising a mixture of such NPs might be capable of exhibiting transparency and slow light effects at optical frequencies. In this section we study the effective electromagnetic properties of a random mixture of such NPs. Also, we study the transparency of a metamaterial composed of coated spherical NPs with excitonic cores and plasmonic shells.

\section{A. Random Mixture of Excitonic and Plasmonic NPs}

We consider a random distribution of silver and $\mathrm{CuCl}$. For calculation of effective electromagnetic parameters of the medium, we use the extended Maxwell Garnett theory for multiphase (or multicomponent) structures in quasi-static limit $[29,30]$, which accounts for dipolar interactions among the NP inclusions [31, 32]:

$$
\varepsilon_{\mathrm{eff}}=\varepsilon_{h} \frac{1+2 \sum_{j=1}^{n} f_{j} \alpha_{j}^{e}}{1-\sum_{j=1}^{n} f_{j} \alpha_{j}^{e}}, \quad \mu_{\mathrm{eff}}=\mu_{h} \frac{1+2 \sum_{j=1}^{n} f_{j} \alpha_{j}^{m}}{1-\sum_{j=1}^{n} f_{j} \alpha_{j}^{m}} .
$$

The sums are over the $n$ components of the medium except the host, $f_{j}$ denotes the volume fraction occupied by the component $j$ (which can be a measure of average distance between the NPs), and $\alpha_{j}^{e}\left(\alpha_{j}^{m}\right)$ is the electric (magnetic) polarizability factor of the component $j$, obtained from the expression -(3/2)i $i a_{1} / x^{3}\left(-(3 / 2) i b_{1} / x^{3}\right)$ [33]. Here, $a_{1}$ and $b_{1}$ are the first Mie coefficients [16]:

$$
\begin{aligned}
& a_{1}=\frac{m \psi_{1}(m x) \psi_{1}^{\prime}(x)-\psi_{1}^{\prime}(m x) \psi_{1}(x)}{\psi_{1}(m x) \xi_{1}^{\prime}(m x)-m \xi_{1}(x) \psi_{1}^{\prime}(m x)}, \\
& b_{1}=\frac{\psi_{1}(m x) \psi_{1}^{\prime}(x)-m \psi_{1}(x) \psi_{1}^{\prime}(m x)}{\psi_{1}(m x) \xi_{1}^{\prime}(m x)-m \xi_{1}(x) \psi_{1}^{\prime}(m x)},
\end{aligned}
$$

where $m=n_{p} / n_{h}$ is the ratio of the particle to host medium refractive indices, $x=2 \pi n_{h} a / \lambda$ is a size parameter, $\psi_{1}(\rho)=$ $\rho j_{1}(\rho)$ and $\xi_{1}(\rho)=\rho h_{1}^{(1)}(\rho)$ are Riccati-Bessel functions proportional to spherical Bessel and Hankel functions.

In Fig. 19, real and imaginary parts of effective dielectric function of a medium is plotted, which contains microscopically random, but macroscopically homogenous distribution of spherical silver and $\mathrm{CuCl}$ NPs with respective diameters and volume fractions of $d_{\mathrm{Ag}}=14 \mathrm{~nm}, d_{\mathrm{CuCl}}=20 \mathrm{~nm}, f_{\mathrm{Ag}}=$ 0.03 and $f_{\mathrm{CuCl}}=0.3$, embedded in a host medium with dielectric constant $\varepsilon_{h}=1.6$. The curve in Fig. 19(a) shows that there is a very steep normal dispersion within the narrow transparency window, corresponding to the narrow dip in the imaginary part of the effective dielectric function of the medium at $\lambda \cong 368.5 \mathrm{~nm}$ [Fig. 19(b)]. Figure 19(b) shows an asymmetric Fano profile with a sharp and strong absorption peak in the vicinity of the narrow transparency dip. 

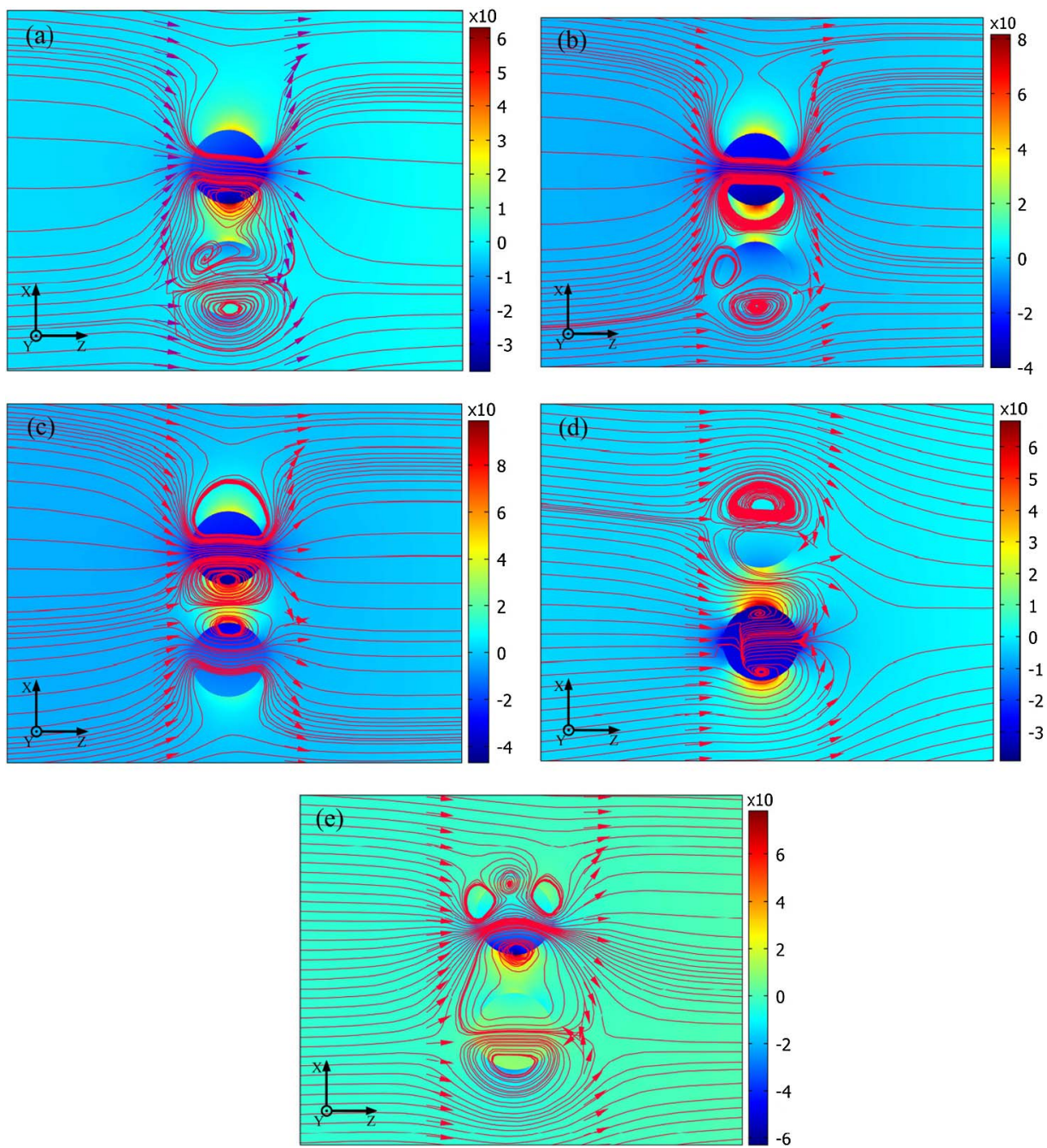

Fig. 18. (Color online) Power flow lines inside and around two coupled silver (lower) and CuCl (upper) NPs with the same parameters and conditions as in Fig. 16, at different wavelengths of (a) $\lambda=368.55 \mathrm{~nm}$, visualizing the mutual coupling of the NPs; (b) $\lambda=368.56 \mathrm{~nm}$, where according to Fig. 17, the total absorption loss is minimum; (c) $\lambda=368.57 \mathrm{~nm}$, corresponding to the minimum value of the electric field at the center of the silver NP (Fig. 16); (d) $\lambda=368.63 \mathrm{~nm}$, corresponding to the maximum point of total absorption loss (Fig. 17), and (e) $\lambda=386.52 \mathrm{~nm}$, near the electric quadrupole resonance peak of the $\mathrm{CuCl} \mathrm{NP}$. The color legends represent the $x$ component of electric field normalized to the incident wave amplitude.

The effective phase index of refraction of the medium is calculated using the relation

$$
n_{\mathrm{eff}}=\sqrt{\left|\varepsilon_{\mathrm{eff}}\right| \cdot\left|\mu_{\mathrm{eff}}\right|} \exp \left(i\left(\theta_{\varepsilon}+\theta_{\mu}\right) / 2\right),
$$

where $\theta_{\varepsilon}$ and $\theta_{\mu}$ are the phases of the $\varepsilon_{\text {eff }}$ and $\mu_{\text {eff }}$, respectively. In the highly dispersive region within the transparency window, the group index of refraction, defined as $n_{g}(\lambda)=n(\lambda)-$ $\lambda[d n(\lambda) / d \lambda]$ (where $n(\lambda)$ is the phase index of refraction as a function of wavelength), can take very high values, which can be utilized in slow light applications. The group index and the imaginary part of the effective phase index as a function of wavelength are shown in Fig. 20, for the same medium parameters as used in Fig. 19. According to Fig. 20, the maximum value of group index, $n_{g}=3740$ (solid curve), corresponds to the value of 0.7 for the imaginary part of phase index (broken curve), and figure of merit (FoM; the ratio of real to imaginary part of phase index) value of $\mathrm{FoM} \cong 1$. Also, the minimum value of the imaginary part of phase index is 0.6 that corresponds to $n_{g}=2300$ and $\mathrm{FoM} \cong 1.6$. Even though that the FoM values are not so pro- mising, we show in the next section that a metamaterial composed of spherical NPs of core-shell geometry can result in much improved FoM values.

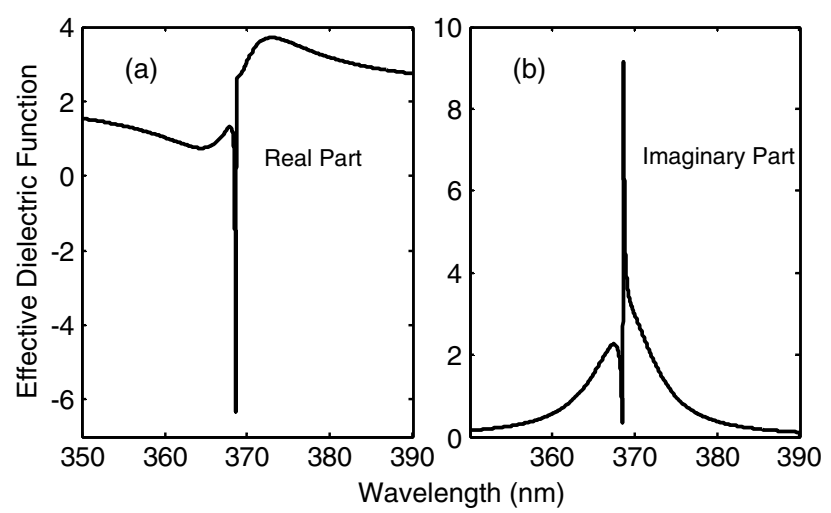

Fig. 19. Real and imaginary parts of the effective dielectric function of a medium containing random distribution of spherical silver and $\mathrm{CuCl} \mathrm{NPs}$ with respective diameters and volume fractions of $d_{\mathrm{Ag}}=14 \mathrm{~nm}, d_{\mathrm{CuCl}}=20 \mathrm{~nm}, f_{\mathrm{Ag}}=0.03$, and $f_{\mathrm{CuCl}}=0.3$, in a host medium with dielectric constant $\varepsilon_{h}=1.6$. 


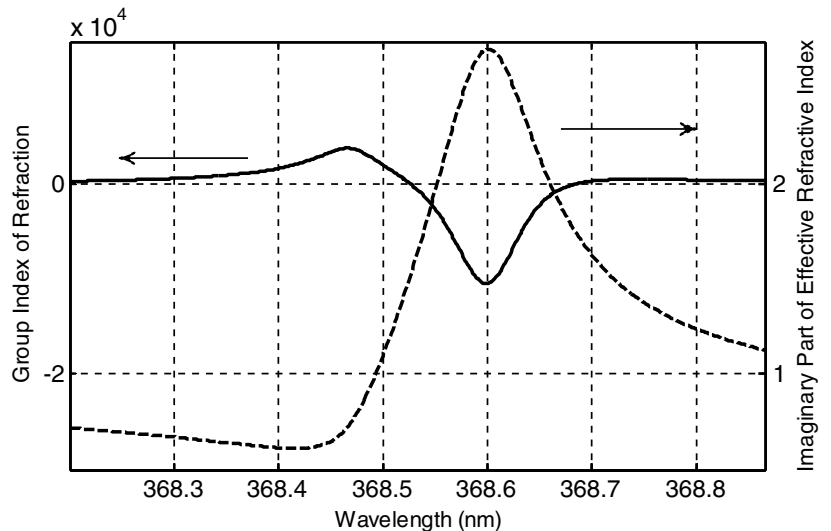

Fig. 20. The group index of refraction and the imaginary part of the effective phase index of refraction as a function of wavelength, for the same medium parameters as in Fig. $\underline{16}$.

The transparency effect in a random mixture of plasmonic and excitonic NPs can be examined numerically by calculating the total absorbed power in the mixture. Such calculations for very large number of NPs usually require very long runtimes, but we have done finite element calculations for a small cluster containing three-dimensional random distribution of 8 silver and $18 \mathrm{CuCl}$ NPs, shown in Fig. 21. The total absorbed power curve is shown in Fig. 22 , which is the result of calculations at 100 discrete wavelengths. In this figure, we see both suppression and enhancement of absorption due to coherent interaction of the NPs. There is also a rather small absorption peak at the right side of the main peak, which can be due to hybridization of the dipolar modes in the cluster. The parameters of the cluster are not optimized for getting maximum transparency. The depth and width of the transparency dip in Fig. 22 can be tuned by changing the filling fraction of the NP components through changing the NPs' dimensions, number density, and the average distance between them.

\section{B. Random Mixture of Spherical Core-Shell NPs}

In this section we show that transparency and slow light effects can also be observed in a medium containing spherical NPs of core-shell geometry with excitonic cores and plasmonic shells. A plasmonic spherical nanoshell has two separate LSP resonances in the optical region, resulting from hybridization of the dipolar modes of a metallic sphere and of a dielectric void in a metallic substrate [34]. We consider metallic nanoshells of aluminum (Al), which by adopting proper

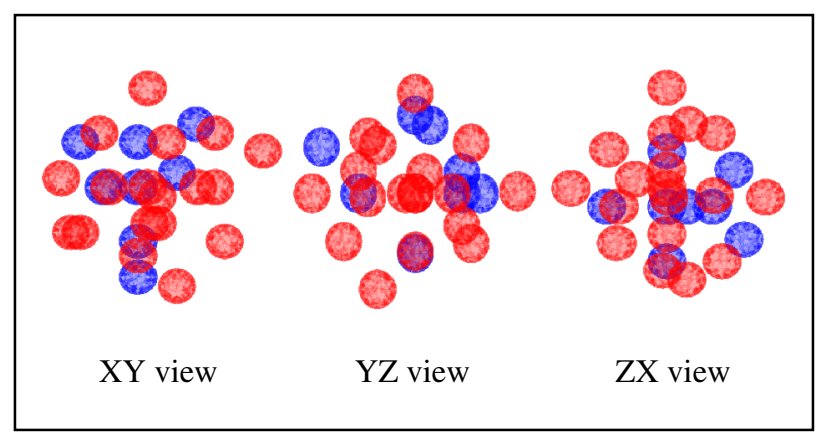

Fig. 21. (Color online) A NP cluster containing three-dimensional random distribution of 8 silver (blue or darker spheres) and $18 \mathrm{CuCl}$ (red spheres) NPs, viewed through $X Y, Y Z$ and $Z X$ planes. All of the NPs have the same radius of $a=10 \mathrm{~nm}$.

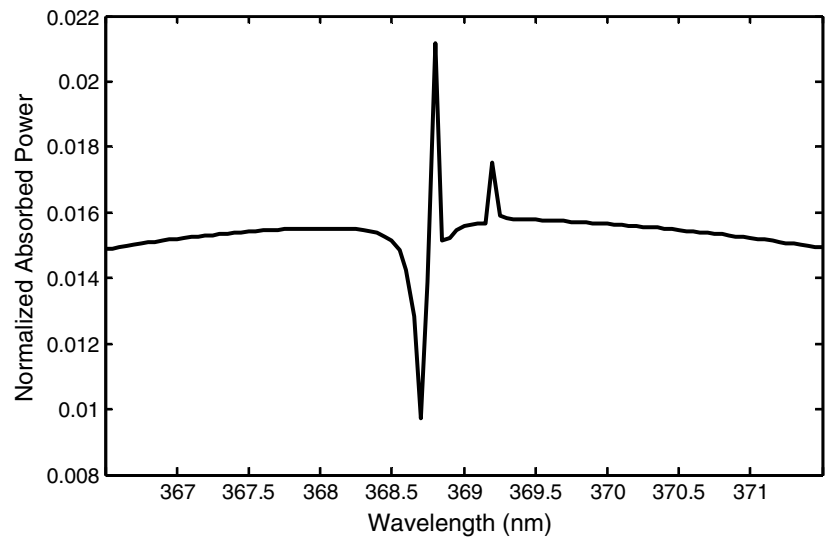

Fig. 22. Normalized (to the incident power) total absorbed power by the cluster of NPs shown in Fig. 21 in a dielectric host material with dielectric constant $\varepsilon_{h}=1.6$, calculated at 100 discrete wavelengths using the finite element method.

radius and thickness and proper dielectric constant of the host material, can provide an LSP resonance in the UV region, coinciding with the excitonic resonance of $\mathrm{CuCl}$ cores. For dielectric function of $\mathrm{Al}$, we use the Drude-Lorentz multioscillator model [35-37]:

$$
\varepsilon_{c}(\omega)=1-\frac{f_{0} \omega_{p}^{2}}{\omega\left(\omega+i \gamma_{0}\right)}+\sum_{i=1}^{5} \frac{f_{i} \omega_{p}^{2}}{\omega_{0 i}^{2}-\omega^{2}-i \gamma_{i} \omega} .
$$

We denote the radius and refractive index of the cores (shells) by $a_{c}$ and $n_{c}\left(a_{s}\right.$ and $\left.n_{s}\right)$, respectively. For evaluation of the effective permittivity and permeability of the medium with core-shell inclusions, we use extended Maxwell Garnett relations:

$$
\begin{aligned}
& \varepsilon_{\text {eff }}=\varepsilon_{h} \frac{1-2 f(3 / 2) i a_{1} / x_{0}^{3}}{1+f(3 / 2) i a_{1} / x_{0}^{3}}, \\
& \mu_{\text {eff }}=\mu_{h} \frac{1-2 f(3 / 2) i b_{1} / x_{0}^{3}}{1+f(3 / 2) i b_{1} / x_{0}^{3}},
\end{aligned}
$$

with Mie coefficients $a_{1}$ and $b_{1}$ given by the following relations (by putting $n=1$ ), derived for core-shell geometry [15]:

$$
a_{n}=\frac{m \psi_{n}(m x)\left[\psi_{n}^{\prime}(x)-A_{n} \chi_{n}^{\prime}(x)\right]-\psi_{n}^{\prime}(m x)\left[\psi_{n}(x)-A_{n} \chi_{n}(x)\right]}{m \xi_{n}(m x)\left[\psi_{n}^{\prime}(x)-A_{n} \chi_{n}^{\prime}(x)\right]-\xi_{n}^{\prime}(m x)\left[\psi_{n}(x)-A_{n} \chi_{n}(x)\right]},
$$

$$
b_{n}=\frac{\psi_{n}(m x)\left[\psi_{n}^{\prime}(x)-B_{n} \chi_{n}^{\prime}(x)\right]-m \psi_{n}^{\prime}(m x)\left[\psi_{n}(x)-B_{n} \chi_{n}(x)\right]}{\xi_{n}(m x)\left[\psi_{n}^{\prime}(x)-B_{n} \chi_{n}^{\prime}(x)\right]-m \xi_{n}^{\prime}(m x)\left[\psi_{n}(x)-B_{n} \chi_{n}(x)\right]},
$$

in which

$$
\begin{aligned}
& A_{n}=\frac{m_{1} \psi_{n}\left(m_{1} x_{1}\right) \psi_{n}^{\prime}\left(x_{1}\right)-\psi_{n}^{\prime}\left(m_{1} x_{1}\right) \psi_{n}\left(x_{1}\right)}{m_{1} \chi_{n}\left(m_{1} x_{1}\right) \psi_{n}^{\prime}\left(x_{1}\right)-\chi_{n}^{\prime}\left(m_{1} x_{1}\right) \psi_{n}\left(x_{1}\right)}, \\
& B_{n}=\frac{\psi_{n}\left(m_{1} x_{1}\right) \psi_{n}^{\prime}\left(x_{1}\right)-m_{1} \psi_{n}^{\prime}\left(m_{1} x_{1}\right) \psi_{n}\left(x_{1}\right)}{\chi_{n}\left(m_{1} x_{1}\right) \psi_{n}^{\prime}\left(x_{1}\right)-m_{1} \chi_{n}^{\prime}\left(m_{1} x_{1}\right) \psi_{n}\left(x_{1}\right)} .
\end{aligned}
$$

Here, $x_{0}=n_{h} k_{0} a_{s}, \quad m=n_{h} / n_{s}, \quad x=n_{s} k_{0} a_{s}, \quad k_{0}=2 \pi / \lambda$, $m_{1}=n_{s} / n_{c}, \quad x_{1}=n_{c} k_{0} a_{c}, \quad \psi_{n}(\rho)=\rho j_{n}(\rho), \quad \chi_{n}(\rho)=\rho y_{n}(\rho)$, 


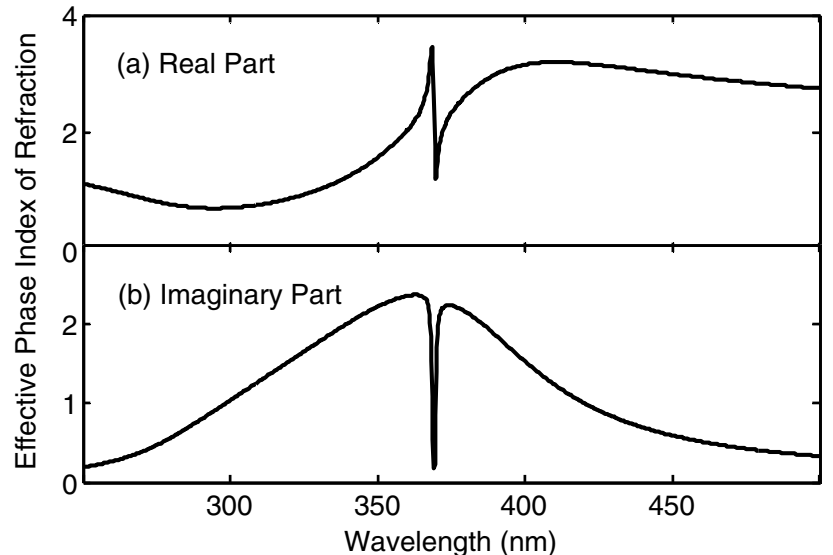

Fig. 23. Real and imaginary parts of phase index of refraction in a medium containing random distribution of core-shell NPs $(\mathrm{CuCl}$ cores and aluminum shells) with volume fraction $f=0.3$ and inner (outer) shell radius of $a_{c}=10 \mathrm{~nm}\left(a_{s}=15 \mathrm{~nm}\right)$, embedded in a host medium with dielectric constant $\varepsilon_{h}=2.3$.

and $\xi_{n}(\rho)=\rho h_{n}^{(1)}(\rho)=\rho\left[j_{n}(\rho)+i y_{n}(\rho)\right]$, in which $j_{n}(\rho)$ and $y_{n}(\rho)$ are Bessel functions of order $n$. Figure 23 shows real and imaginary parts of phase index of refraction, in a medium containing random distribution of core-shell NPs with volume fraction $f=0.3$ and inner (outer) shell radius of $a_{c}=10 \mathrm{~nm}$ $\left(a_{s}=15 \mathrm{~nm}\right)$, embedded in a host with dielectric constant $\varepsilon_{h}=2.3$. The highly dispersive region in this figure within the narrow transparency window occurs around the wavelength of $\lambda=369 \mathrm{~nm}$.

The group index and the imaginary part of the phase index, $n_{\text {eff }}$, are shown in Fig. 24, where the group index takes the value of $n_{g} \cong 1300$, at the wavelength of $\lambda=369.2$, corresponding to the imaginary part of phase index $\cong 0.13$ and FoM value of $\cong 17$, which shows nearly 1 order of magnitude improvement compared to the case of random mixture of NPs in the previous section.

An important parameter for evaluation of the performance of slow light structures is delay-bandwidth product given by $\Delta \tau . B=\sqrt{\ln 2} \sqrt{L \Delta \alpha(0)}$ [38], in which $L$ is the slow light device length and $\Delta \alpha(0)$ is the depth of the spectral hole defined as the difference between the original and modified absorption spectra at the center frequency. Calculating the depth of spectral hole in two different cases, we see that for a single wavelength propagation length, the delay-bandwidth product

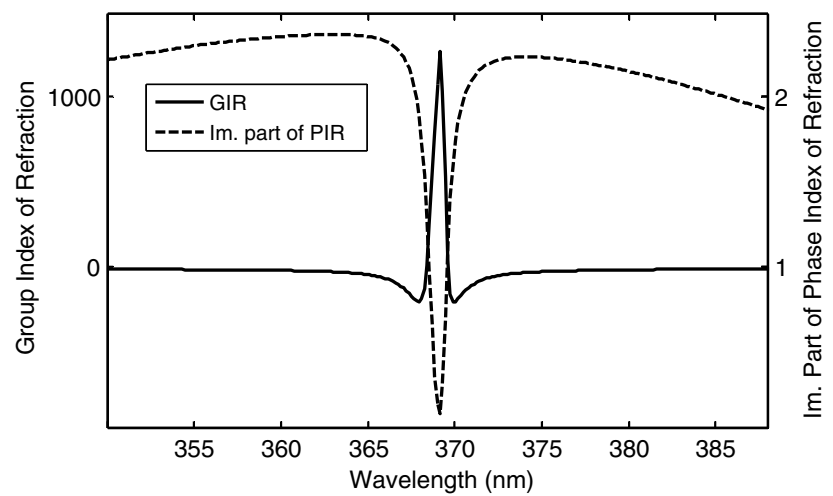

Fig. 24. The group index of refraction (GIR) and the imaginary part of the phase index of refraction (PIR), for the same medium described in Fig. 23. is equal to 6.1 in the case of random mixture of $\mathrm{CuCl}$ and $\mathrm{Ag}$ nanoparticles (in the condition described in Fig. 19), and it is equal to 3.4 in the case of random distribution of coated nanoparticles with $\mathrm{CuCl}$ cores and aluminum shells (with parameters given in Fig. 23). The delay-bandwidth product values are higher than that obtained in [39] and are comparable to those obtained in $[\underline{40,41]}$.

\section{SUMMARY}

The classical interactions of plasmonic and excitonic NPs can result in enhanced or suppressed absorption of electromagnetic waves. The suppressed absorption or transparency is at the same spectral region of steep variations in real part of the plasmonic-excitonic pair's polarizability, which can result in high normal dispersions in metamaterials composed of such coupled NPs. It is shown that within the transparency window, group indices of refraction of the order of several thousands can be achieved in metamaterials composed of random distribution of excitonic (copper chloride) and plasmonic (silver) NPs, or random distribution of core-shell NPs with copper chloride cores and aluminum shells in the UV region.

\section{ACKNOWLEDGMENTS}

We acknowledge the metamaterials group in nanophotonics cluster at DTU Fotonik, especially Dr. Andrey Novitsky and Dr. Andrei Andryieuski for their helpful comments and ideas.

\section{REFERENCES}

1. G. P. Wiederrecht, G. A. Wurtz, and J. Hranisavljevic, "coherent coupling of molecular excitons to electronic polarizations of noble metal nanoparticles," Nano Lett. 4, 2121-2125 (2004).

2. N. T. Fofang, T.-H. Park, O. Neumann, N. A. Mirin, P. Nordlander, and N. J. Halas, "Plexcitonic nanoparticles: plasmon-exciton coupling in nanoshell-J-aggregate complexes," Nano Lett. 8, 34813487 (2008).

3. N. T. Fofang, N. K. Grady, Z. Fan, A. O. Govorov, and N. J. Halas, "Plexciton dynamics: exciton plasmon coupling in a $J$-aggregate Au nanoshell complex provides a mechanism for nonlinearity," Nano Lett. 11, 1556-1560 (2011).

4. A. O. Govorov, G. W. Bryant, W. Zhang, T. Skeini, J. Lee, N. A. Kotov, J. M. Slocik, and R. R. Naik, "Exciton-plasmon interaction and hybrid excitons in semiconductor-metal nanoparticle assemblies," Nano Lett. 6, 984-994 (2006).

5. W. Zhang, A. O. Govorov, and G. W. Bryant, "Semiconductormetal nanoparticle molecules: hybrid excitons and the nonlinear Fano effect," Phys. Rev. Lett. 97, 146804 (2006).

6. R. D. Artuso and G. W. Bryant, "Optical response of strongly coupled quantum dot-metal nanoparticle systems: double peaked Fano structure and bistability," Nano Lett. 8, 21062111 (2008).

7. S. M. Sadeghi, L. Deng, X. Li, and W.-P. Huang, "Plasmonic (thermal) electromagnetically induced transparency in metallic nanoparticle-quantum dot hybrid systems," Nanotechnology $\mathbf{2 0}$, 365401 (2009).

8. A. Manjavacas, F. J. García de Abajo, and P. Nordlander, "Quantum plexcitonics: strongly interacting plasmons and excitons," Nano Lett. 11, 2318-2323 (2011).

9. V. Yannopapas and N. V. Vitanov, "Photoexcitation-induced magnetism in arrays of semiconductor nanoparticles with a strong excitonic oscillator strength," Phys. Rev. B 74, 193304 (2006).

10. V. Yannopapas, "Negative refractive index in the near-UV from Au-coated $\mathrm{CuCl}$ nanoparticle superlattices," Phys. Stat. Sol. (RRL) 1, 208-210 (2007).

11. A.-G. Kussow, A. Akyurtlu, and N. Angkawisittpanet, "Optically isotropic negative index of refraction metamaterial," Phys. Stat. Sol. (b) 245, 992-997 (2008).

12. V. Agranovich and V. Ginzburg, Crystal Optics with Spatial Dispersion and Excitons, 2nd ed. (Springer, 1984). 
13. S. Chesi, M. Artoni, G. C. La Rocca, F. Bassani, and A. Mysyrowicz, "Polaritonic stop-band transparency via exciton-biexciton Coupling in CuCl," Phys. Rev. Lett. 91, 057402 (2003).

14. M. Artoni, G. La Rocca, and F. Bassani, "Resonantly absorbing one-dimensional photonic crystals," Phys. Rev. E 72, 046604 (2005).

15. C. F. Bohren and D. R. Huffman, Absorption and Scattering of Light by Small Particles (Wiley, 1983).

16. A. Panahpour and H. Latifi, "Electromagnetic transparency and slow light in an isotropic 3D optical metamaterial, due to Fanolike coupling of Mie resonances in excitonic nano-sphere inclusions," Opt. Commun. 284, 1701-1710 (2011).

17. V. P. Drachev, U. K. Chettiar, A. V. Kildishev, H.-K. Yuan, W. Cai, and V. M. Shalaev, "The Ag dielectric function in plasmonic metamaterials," Opt. Express 16, 1186-1195 (2008).

18. P. B. Johnson and R. W. Christy, "Optical constants of the noble metals,” Phys. Rev. B 6, 4370-4379 (1972).

19. U. Kreibig, "Electronic properties of small silver particles: the optical constants and their temperature dependence," J. Phys. F 4, 999-1014 (1974).

20. S. Zou, N. Janel, and G. C. Schatz, "Silver nanoparticle array structures that produce remarkably narrow plasmon lineshapes,” J. Chem. Phys. 120, 10871-10876 (2004).

21. M. Fleischhauer, A. Imamoglu, and J. P. Marangos, "Electromagnetically induced transparency: Optics in coherent media," Rev. Mod. Phys. 77, 633-673 (2005).

22. S. Zhang, D. A. Genov, Y. Wang, M. Liu, and X. Zhang, "Plasmoninduced transparency in metamaterials," Phys. Rev. Lett. 101, 047401 (2008)

23. N. Liu, L. Langguth, T. Weiss, J. Kästel, M. Fleischhauer, T. Pfau, and H. Giessen, "Plasmonic analogue of electromagnetically induced transparency at the Drude damping level," Nat. Mater. 8, 758-762 (2009).

24. S.-Y. Chiam, R. Singh, C. Rockstuhl, F. Lederer, W. Zhang, and A. A. Bettiol1, "Analogue of electromagnetically induced transparency in a terahertz metamaterial," Phys. Rev. B 80, 153103 (2009).

25. P. Tassin, L. Zhang, Th. Koschny, E. N. Economou, and C. M. Soukoulis, "Low-loss metamaterials based on classical electromagnetically induced transparency," Phys. Rev. Lett. 102 , 053901 (2009).

26. P. Tassin, L. Zhang, T. Koschny, E. N. Economou, and C. M. Soukoulis, "Planar designs for electromagnetically induced transparency in metamaterials," Opt. Express 17, 5595-5605 (2009).
27. Y. S. Joe, A. M. Satanin, and C. S. Kim, "Classical analogy of Fano resonances,” Phys. Scr. 74, 259-266 (2006).

28. M. V. Bashevoy, V. A. Fedotov, and N. I. Zheludev, "Optical whirlpool on an absorbing metallic nanoparticle," Opt. Express 13, 8372-8379 (2005).

29. A. Sihvola, "Mixing rules with complex dielectric coefficients," Subsurf. Sens. Technol. Appl. 1, 393-415 (2000)

30. Q. Wang, D. Tiana, G. Xionga, and Z. Zhoua, "A simplified model for the dielectric function of three-component composite materials," Physica A 275, 256-261 (2000).

31. P. Mallet, C. A. Guérin, and A. Sentenac, "Maxwell Garnett mixing rule in the presence of multiple scattering: derivation and accuracy," Phys. Rev. B 72, 014205 (2005).

32. C. F. Bohren and N. C. Wickramasinghe, "On the computation of optical properties of heterogeneous grains," Astrophys. Space Sci. 50, 461-472 (1977).

33. W. T. Doyle, "Optical properties of a suspension of metal spheres," Phys. Rev. B 39, 9852-9858 (1989).

34. S. A. Maier, Plasmonics: Fundamentals and Applications (Springer, 2007).

35. A. D. Rakic, A. B. Djurišic, J. M. Elazar, and M. L. Majewski, “Optical properties of metallic films for vertical-cavity optoelectronic devices," Appl. Opt. 37, 5271-5283 (1998).

36. M. I. Markovic and A. D. Rakic, "Determination of reflection coefficients of laser light of Wavelength $\lambda \in(0.22 \mu \mathrm{m}, 200 \mu \mathrm{m})$ from the surface of aluminum using the Lorentz-Drude model," Appl. Opt. 29, 3479-3483 (1990).

37. M. I. Markovic and A. D. Rakic, "Determination of optical properties of aluminum including electron reradiation in the LorentzDrude model," Opt. Laser Technol. 22, 394-398 (1990).

38. P. C. Ku, C. J. Chang-Hasnain, and S. L. Chuang, "Slow light in semiconductor heterostructures," J. Phys. D 40, R93-R107 (2007).

39. C. Kurter, P. Tassin, L. Zhang, T. Koschny, A. P. Zhuravel, A. V. Ustinov, S. M. Anlage, and C. M. Soukoulis, "Classical analogue of electromagnetically induced transparency with a metalsuperconductor hybrid metamaterial," Phys. Rev. Lett. 107, 043901 (2011).

40. P. Tassin, L. Zhang, Th. Koschny, E. N. Economou, and C. M Soukoulis, "Low-loss metamaterials based on classical electromagnetically induced transparency," Phys. Rev. Lett. 102 053901 (2009).

41. R. D. Kekatpure, E. S. Barnard, W. Cai, and M. L. Brongersma "Phase-coupled plasmon-induced transparency," Phys. Rev. Lett. 104, 243902 (2010). 